\title{
EVALUACIÓN DE MERCADOS PARA EL FRIJOL CENTROAMERICANO EN COMUNIDADES ÉTNICAS DE EE.UU. ${ }^{1}$
}

\author{
Miguel Zamora ${ }^{2}$, Richard Bernsten ${ }^{2}$
}

\begin{abstract}
RESUMEN
Evaluación de mercados para el frijol centroamericano en comunidades étnicas de EE.UU. Este documento presenta información sobre el mercado étnico del frijol centroamericano en EE.UU., describe las oportunidades y dificultades potenciales para estos países, y ofrece información a exportadores potenciales en estos países para aminorar estas dificultades. No existen estudios que describan la demanda étnica potencial por frijol centroamericano en EE.UU. Se determinaron los estados y ciudades con las más altas concentraciones de personas de origen centroamericano en EE.UU. Los costarricenses y panameños no fueron incluidos en este estudio por tener poblaciones relativamente pequeñas en EE.UU. Chicago, Miami, Los Ángeles, San Francisco y Washington D.C fueron visitadas para: a) recoger datos sobre la disponibilidad de frijol en tiendas detallistas, b) entrevistar consumidores centroamericanos acerca de sus preferencias de frijol, c) entrevistar organizaciones centroamericanas, distribuidores e importadores de frijol para entender la dinámica del comercio del frijol. Comerciantes estadounidenses también estaban importando frijol de Honduras. Los frijoles centroamericanos se venden al detalle a precios entre $25-50 \%$ mayores que otros frijoles similares. Además, los centroamericanos en EE.UU. prefieren fuertemente los frijoles de su país.
\end{abstract}

Palabras claves: mercados étnicos, frijol, Centro América, EE.UU.

\begin{abstract}
An assessment of markets for Central American dry beans in USA ethnic communities. This paper presents information about the U.S. ethnic market for dry beans of Central American origin, describes the potential opportunities and constraints that these four countries face in expanding bean exports, and provides information to policymakers and potential exporters in these countries regarding ways to relax these constraints. No study has been carried out to describe the potential ethnic demand in the USA for Central American dry beans. USA Census Bureau data were analyzed to identify the states and cities with the highest concentration of people from these countries. Five cities were identified and visited in order to: a) obtain data on the availability of beans in retail stores, b) survey Central American consumers on their dry beans preferences, c) survey Central American organizations, importers, and distributors to understand the dynamics of the dry bean trade. Traders in Miami are also importing dry beans from Honduras. Central American dry beans sell at a price $25-50 \%$ higher than USA beans of similar market classes. Furthermore, people of Central American origin have a strong preference for dry beans from their country of origin.
\end{abstract}

Key words: ethnic markets, beans, Central América, USA.

\section{INTRODUCCIÓN}

El mercado hispano en EE.UU. es un mercado importante para productos comestibles, especialmente para los frijoles. Según el Food Marketing Institute, los hogares de origen hispano gastan en promedio $\$ 117.00$ dólares por semana en comestibles, comparado con el hogar promedio de EE.UU. que gasta $\$ 87.00$ por semana. Los hispanos visitan las abarroterías un promedio de 4,7 veces por semana, comparado con las 2,2 visitas por semana que realiza el estadounidense promedio. Además, el 85\% de los alimentos consumidos en casa por los hogares hispanos es comida hispana (Food Marketing Institute 2002). Humphreys, en informe para el

1 Recibido para publicación el 2 de junio del 2004. Trabajo presentado durante la L Reunión del PCCMCA, 19- 23 de Abril, 2004, San Salvador, El Salvador.

2 Departamento de Economía Agrícola, Michigan State University, East Lansing MI 48833, EE.UU. (colaboración en la edición de J.C. Rosas, Escuela Agrícola Panamericana, Zamorano, Honduras). Correo electrónico: zamoram3@msu.edu. 
Centro Selig para el Crecimiento Económico en la Universidad de Georgia, estimó que en el 2001 el poder de compra hispano en EE.UU. era aproximadamente $\$ 452$ mil millones de dólares, y además que aumentaría a $\$$ 653 mil millones en el 2003, y a \$1,014 mil millones en el 2008 (Humphreys 2003). Este crecimiento en poder de compra se proyecta a ser relativamente mayor que el de cualquier otro grupo étnico en los EE.UU. Esto puede implicar que el mercado hispano crecerá en valor e importancia en los futuros años.

Lucier et al. (2000), usando datos de la Encuesta Continua de Tomas de Alimento por Individuos, calculan que en un día dado, casi $14 \%$ de los estadounidenses consumen por lo menos una comida que contiene frijoles cocinados. La mayoría de esos frijoles se prepara en el hogar del consumidor. Las personas de origen hispano consumen proporcionalmente más frijoles cocinados que cualquier otro grupo étnico en EE.UU. Mientras los hispanos representaron alrededor del $11 \%$ de la población de EE.UU. en 1996, estos consumieron el 33\% de los frijoles (Lucier et al. 2000).

Los consumidores centroamericanos prefieren frijoles con características específicas de sabor, tiempo de cocción y color. En una encuesta realizada a 203 hogares de origen salvadoreño en Houston y Los Ángeles, se encontró que alrededor del $89 \%$ de estos hogares consumió cerca de $1,4 \mathrm{~kg}$ de frijoles rojos en una semana cualquiera en el 2001 (Batres-Márquez, Jensen y Brester 2001). Los autores encontraron también que el $58,6 \%$ de las hogares salvadoreños inspeccionados, consumirían hasta $1,7 \mathrm{~kg}$ de frijoles rojos salvadoreños si estos frijoles estuvieran disponibles para la compra. Además, en el 2003, el gobierno salvadoreño estimó que el mercado potencial para los frijoles salvadoreños en EE.UU. era alrededor de $\$ 4.8$ millones de dólares (Ministerio de Economía de El Salvador 2003). Sin embargo, esta cantidad se basó en un estimado de la población salvadoreña en EE.UU. que era apreciablemente más alto que el número informado por el censo de EE.UU. del año 2000.

\section{Objetivo}

Para esta evaluación de mercados para los frijoles centroamericanos en EE.UU. se requiere: 1) documentar la distribución geográfica y preferencias de los consumidores centroamericanos en EE.UU.; 2) documentar el nivel actual de importaciones estadounidenses de frijol de Centro América y los canales de comercialización de este frijol; y 3) describir las limitaciones y oportunidades para aumentar las exportaciones de frijol a las comunidades centroamericanas en los EE.UU.

\section{Limitaciones del estudio}

La limitación más grande en este estudio es el número real de encuestas que se realizaron a consumidores. Fue muy difícil, logísticamente hablando, alcanzar un número más grande de consumidores encuestados. Esta limitación se podría reducir, en estudios futuros parecidos, trabajando de cerca con las embajadas y consulados centroamericanos para obtener un número mayor de encuestas a consumidores.

\section{METODOLOGÍA}

\section{La concentración geográfica de poblaciones cen- troamericanas en EE.UU.}

Datos del Censo del 2000 de EE.UU. fueron analizados y se rastreó los estados y las ciudades con las concentraciones más altas de centroamericanos para tratar de ubicar las tiendas latinas locales que venden frijoles. Empezando con los estados que tienen la concentración más alta de centroamericanos, se escogieron los condados en estos estados y las ciudades en estos condados, según los mismos criterios. Este rastreo se efectuó seleccionando las áreas (conocidas en inglés como "tracts") ${ }^{1}$ con mayor concentración de centroamericanos. Estas áreas en el Censo 2000 son muy específicas, y permitieron la localización de calles y vecindarios dentro de estas ciudades. Las ciudades que tuvieron las áreas con la concentración más alta de centroamericanos fueron visitadas para recolectar datos. Se utilizó el programa ArcView ${ }^{\circledR}$ para trabajar con datos del GIS (sistema geográfico de información) y trazar los condados, las ciudades, las áreas y las calles con las concentraciones más altas de centroamericanos en los EE.UU., usando los datos del censo 2000. Estos mapas se utilizaron en los viajes para identificar y caminar en las áreas escogidas, y de esta manera localizar las abarroterías hispanas y las organizaciones que trabajan ayudando a los centroamericanos en EE.UU. También se visitaron las embajadas y algunos consulados de cuatro países de Centro América para reunir información acerca de estas organizaciones que trabajan con personas de origen centroamericano. En este estudio los términos Centro América y centroamericano incluyen a El Salvador, Guatemala, Honduras y Nicaragua únicamente.

\footnotetext{
1 Un tract es "una subdivisión estadística, relativamente permanente y pequeña, de un condado delineado por un comité local de usuarios del censo para el propósito de presentar los datos". Estos tracts contienen las calles en un área promedio aproximada de 1 milla cuadrada, aunque el área real puede variar (Oficina del Censo de EE.UU. 2000).
} 


\section{Comercio de frijoles entre países de América central y EE.UU.}

Se analizó información secundaria para obtener datos sobre las importaciones y exportaciones estadounidenses de frijol de y hacia Centro América. Datos provenientes de la base de datos USA trade on-line (Comercio de EE.UU. en linea) se analizaron para valorar estas importaciones y exportaciones y sus tendencias.

\section{Preferencias de consumidores y tendencias}

Se contactaron a las organizaciones centroamericanas para obtener información sobre las tiendas de abarroterías hispanas y comerciantes de alimentos en las diferentes ciudades visitadas. Varias abarroterías étnicas con una proporción alta de centroamericanos, fueron visitadas para reunir información acerca del origen y precios de los frijoles disponibles para la venta. Se condujeron encuestas a consumidores en varias embajadas, consulados y centros de centroamericanos en Chicago, Los Ángeles, Washington D.C., Miami y San Francisco. Además se visitó la convención Expo-comida Latina en Los Ángeles donde se encuestaron varios importadores y distribuidores.

\section{RESULTADOS Y DISCUSIÓN}

\section{La concentración geográfica de los centroamerica- nos en los EE.UU.}

El Cuadro 1 muestra el aumento en el número de hispanos y centroamericanos en EE.UU. En la última década, la población hispana aumentó en 57,9\%, yendo de 22,4 millones en 1990 a 35,3 millones en el 2000, comparado con un aumento de sólo $13,1 \%$ para la población total de EE.UU.

Los centroamericanos representaron solo el $0,60 \%$ del total de personas del Censo 2000. Los centroamericanos aumentaron en un 27,4\% de 1990 al 2000; sin embargo, la estimación en el $2001^{2}$ muestra un aumento del $37,8 \%$ comparado con los datos del censo del año 2000. Los salvadoreños son el grupo más grande de centroamericanos en los EE.UU., representando aproximadamente el 39\% del total de centroamericanos. Los guatemaltecos representan el 22,1\%, los nicaragüenses el 10,5\% y los hondureños el $12,9 \%$, del total de centroamericanos en el Censo 2000.

Según la Oficina del Censo de los EE.UU, la mitad de todas las personas de origen hispano vive en sólo dos

Cuadro 1. Número de personas de origen hispano y centroamericano en EE.UU. Censos de 1990 y del 2000 y estimados del 2001.

\begin{tabular}{|c|c|c|c|c|c|}
\hline \multirow[b]{2}{*}{ Grupo Poblacional } & \multicolumn{4}{|c|}{ Población en Censo (número de habitantes) } & \multirow{2}{*}{$\begin{array}{l}\text { Estimado en base a encuesta } \\
2001\end{array}$} \\
\hline & 1990 & 2000 & $\begin{array}{c}\text { Total de CA en } \\
\text { el } 2000(\%)\end{array}$ & $\begin{array}{c}\text { Incremento de } \\
1990 \text { al } 2000(\%)\end{array}$ & \\
\hline Total: & 248709.873 & 281421.906 & & 13,2 & 277017.622 \\
\hline No-Hispano & 226809.784 & 246116.088 & & 8,5 & 240816.841 \\
\hline Hispano & 22354.059 & 35305.818 & & 58,0 & 36200.781 \\
\hline Centro- Americano: & 1323.830 & 1686.937 & 100,0 & 27,4 & 2325.600 \\
\hline Costarricense & & 68.588 & 4,1 & & 89.842 \\
\hline Guatemalteco & 268.779 & 372.487 & 22,1 & 38,6 & 518.233 \\
\hline Hondureño & 131.066 & 217.569 & 12,9 & 66,0 & 307.346 \\
\hline Nicaragüense & 202.658 & 177.684 & 10,5 & $-12,3$ & 268.961 \\
\hline Panameño & 92.013 & 91.723 & 5,4 & $-0,3$ & 106.909 \\
\hline Salvadoreño & 565.081 & 655.165 & 38,8 & 15,9 & 967.148 \\
\hline Otros CA* & 64.233 & 103.721 & 6,1 & 61,5 & 67.161 \\
\hline
\end{tabular}

* "Otros CA" representa el número de personas que declararon que eran de origen centroamericano en el Censo 2000, pero que no declararon ningún país específico de origen.

Fuente: Oficina del Censo de EE.UU.

\footnotetext{
2 Proveniente del universo de la Encuesta suplementaria del 2001. Esta inspección es limitada a la población del hogar y excluye a la población que vive en instituciones, en dormitorios colegiales, y en otros cuartos del grupo. Los datos se basan en una muestra y son susceptibles a variabilidad muestral (Oficina del Censo de EE.UU. 2000).
} 
estados: California y Texas; sin embargo, la distribución de centroamericanos es un poco diferente. En los estados de California, Florida y New York vive el 56\% de los centroamericanos en EE.UU. En el Cuadro 2 se indican los estados con la proporción más alta de centroamericanos en los EEUU. Estos 14 estados justifican casi el $90 \%$ de los centroamericanos en los EE.UU., según el Censo 2000.

Las ciudades de Los Ángeles, New York y Miami, representan los lugares con la concentración más alta de centroamericanos por país de origen específico. Aún cuando Houston tiene más centroamericanos que Miami, Miami tiene más nicaragüenses que cualquier otra ciudad en los EEUU. Sin embargo, el mayor número de guatemaltecos, costarricenses, hondureños, panameños y salvadoreños está en Los Ángeles y New York. Washington D.C. y las localidades circundantes (Arlington y Alexandria) tienen una concentración alta de centroamericanos en zonas específicas de la ciudad.

El Cuadro 3 muestra a las ciudades de Miami y Hialeah como un solo lugar (Miami), y a las ciudades de Washington D.C., Arlington y Alexandria como un sólo lugar también (Washington) debido a su proximidad geográfica. Los Ángeles, New York y Miami son los lugares con las poblaciones más grandes de guatemaltecos, hondureños, nicaragüenses y salvadoreños.

Considerando que Los Ángeles, Miami y Washington tienen el porcentaje más alto de centroamericanos como proporción de su población total, se visitaron esas

Cuadro 2. Población y porcentaje de personas de origen centroamericano en EE.UU. en los estados con la mayor concentración de centroamericanos (CANs). 2000.

\begin{tabular}{|c|c|c|c|c|c|c|c|c|c|}
\hline \multirow[b]{2}{*}{ Estado } & \multirow{2}{*}{$\begin{array}{c}\text { Población } \\
\text { de CANs }\end{array}$} & \multicolumn{2}{|c|}{ Guatemaltecos } & \multicolumn{2}{|c|}{ Hondureños } & \multicolumn{2}{|c|}{ Nicaragüenses } & \multicolumn{2}{|c|}{ Salvadoreños } \\
\hline & & Total & $\% *$ & Total & $\% *$ & Total & $\% *$ & Total & $\% *$ \\
\hline California & 576.330 & 143.500 & 39,1 & 30.372 & 14,1 & 51.336 & 29,0 & 272.999 & 41,8 \\
\hline Florida & 202.772 & 28.650 & 7,8 & 41.229 & 19,1 & 79.559 & 44,9 & 20.701 & 3,2 \\
\hline New York & 181.875 & 29.074 & 7,9 & 35.135 & 16,3 & 8,033 & 4,5 & 72.713 & 11,1 \\
\hline Texas & 146.723 & 18.539 & 5,0 & 24.179 & 11,2 & 7.487 & 4,2 & 79.204 & 12,1 \\
\hline New Jersey & 80.497 & 16.992 & 4,6 & 15.431 & 7,1 & 4.384 & 2,5 & 25.230 & 3,9 \\
\hline Virginia & 73.140 & 10.000 & 2,7 & 7.819 & 3,6 & 3.191 & 1,8 & 43.653 & 6,7 \\
\hline Maryland & 56.135 & 8.304 & 2,3 & 4.067 & 1,9 & 3.440 & 1,9 & 34.433 & 5,3 \\
\hline Illinois & 39.377 & 19.790 & 5,4 & 5.992 & 2,8 & 1.500 & 0,8 & 7.085 & 1,1 \\
\hline Massachusetts & 38.317 & 11.437 & 3,1 & 5.125 & 2,4 & 697 & 0,4 & 15.900 & 2,4 \\
\hline Georgia & 31.813 & 10.718 & 2,9 & 5.158 & 2,4 & 1.394 & 0,8 & 8.497 & 1,3 \\
\hline North Carolina & 30.783 & 5.966 & 1,6 & 8.321 & 3,9 & 1.449 & 0,8 & 8.679 & 1,3 \\
\hline Nevada & 18.747 & 4.106 & 1,1 & 1.316 & 0,6 & 1.583 & 0,9 & 9.386 & 1,4 \\
\hline Louisiana & 17.327 & 2.093 & 0,6 & 8.792 & 4,1 & 2.804 & 1,6 & 1.127 & 0,2 \\
\hline District of Columbia & 15.803 & 1.350 & 0,4 & 853 & 0,4 & 594 & 0,3 & 11.741 & 1,8 \\
\hline Total de 14 estados & 1509.639 & 310.519 & 84,5 & 193.789 & 89,8 & 167.451 & 94,6 & 611.348 & 93,7 \\
\hline
\end{tabular}

*Porcentaje dentro del total de cada país.

Fuente: Oficina del Censo de EE.UU.

Cuadro 3. Poblaciones y porcentajes de personas de origen centroamericano en ciudades más pobladas por centroamericanos (CANs) en EE.UU., 2000

\begin{tabular}{|c|c|c|c|c|c|c|c|c|c|c|c|}
\hline \multirow[b]{2}{*}{ Ciudad } & \multirow{2}{*}{$\begin{array}{c}\text { Población } \\
\text { Total }\end{array}$} & \multicolumn{2}{|c|}{ CANs } & \multicolumn{2}{|c|}{ Guatemaltecos } & \multicolumn{2}{|c|}{ Hondureños } & \multicolumn{2}{|c|}{ Nicaragüienses } & \multicolumn{2}{|c|}{ Salvadoreños } \\
\hline & & Total & $\%$ & Total & $\%$ & Total & $\%$ & Total & $\%$ & Total & $\%$ \\
\hline Los Angeles & 3694.820 & 238.191 & 6,45 & 65.992 & 1,78 & 12.030 & 0,33 & 8.792 & 0,24 & 126.197 & 3,42 \\
\hline New York & 8008.278 & 99.099 & 1,24 & 15.212 & 0,19 & 25.600 & 0,32 & 6.451 & 0,08 & 24.516 & 0,31 \\
\hline Houston & 1953.631 & 60.642 & 3,10 & 7.220 & 0,37 & 10.284 & 0,53 & 2.196 & 0,11 & 36.799 & 1,88 \\
\hline Miami & 588.889 & 54.826 & 9,31 & 3.298 & 0,56 & 14.711 & 2,50 & 29.754 & 5,05 & 3.245 & 0,55 \\
\hline Washington & 889.795 & 34.367 & 3,86 & 3.694 & 0,42 & 2.865 & 0,32 & 1.315 & 0,15 & 23.848 & 2,68 \\
\hline San Francisco & 776.733 & 23.367 & 3,01 & 3.196 & 0,41 & 934 & 0,12 & 5.459 & 0,70 & 10.655 & 1,37 \\
\hline Chicago & 2896.016 & 23.39 & 0,81 & 13.610 & 0,47 & 3.049 & 0,11 & 778 & 0,03 & 3.468 & 0,12 \\
\hline Dallas & 1188.580 & 14.972 & 1,26 & 1.950 & 0,16 & 2.637 & 0,22 & 407 & 0,03 & 8.582 & 0,72 \\
\hline
\end{tabular}

Fuente: Oficina del Censo de EE.UU. 
ciudades para obtener información acerca del mercado potencial para frijoles de esos países. San Francisco se escogió también debido a la alta concentración de centroamericanos en áreas específicas de la ciudad y al alto número de nicaragüenses. Chicago se escogió por su proximidad a la Universidad del Estado de Michigan y al número de guatemaltecos y hondureños en esta ciudad.

\section{Importaciones y exportaciones de frijol}

Mientras las exportaciones de frijol estadounidense a Centro América han variado mucho, las importa- ciones totales de EE.UU provenientes de esta región han estado aumentando constantemente en los últimos cuatro años (Figura 1).

Los Ángeles, Miami, New York y Houston son los puertos de entrada más importantes para los frijoles centroamericanos (Cuadro 4). Los cuatro puertos recibieron alrededor del $94 \%$ del total de importaciones de frijoles centroamericanos. Miami es el más importante de estos puertos, recibiendo más del $40 \%$ del total de frijoles centroamericanos en el 2003.

En la última década, EE.UU. ha exportado más frijoles a Guatemala y Honduras de lo que EE.UU ha
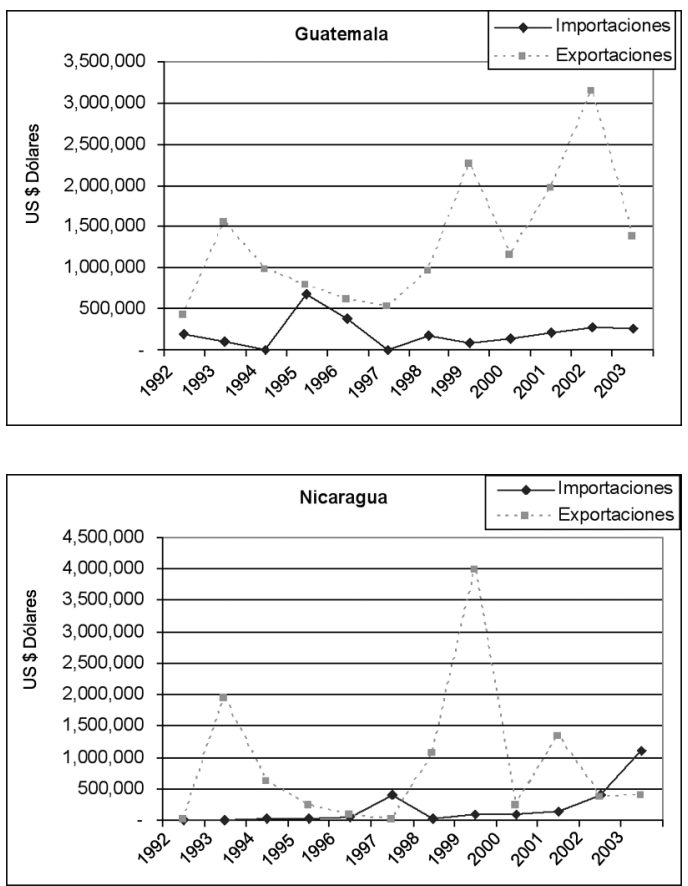

Figura 1. Valor en dólares de las importaciones y exportaciones estadounidenses de frijol proveniente de El Salvador, Guatemala, Honduras y Nicaragua, 1992-2003. Fuente: USA Trade on-line.

Cuadro 4. Valor en dólares de las importaciones estadounidenses de frijol centroamericano enviado a puertos seleccionados dentro de EE.UU., 2002 y 2003.

\begin{tabular}{lrrrrrr}
\hline Puerto de entrada & \multicolumn{2}{c}{ Enero a diciembre, 2003 } & & \multicolumn{2}{c}{ Enero a diciembre, 2002 } \\
\cline { 2 - 3 } \cline { 5 - 6 } & Valor total (US\$) & \% de total* & & Valor total (US\$) & \% de total* \\
\hline \multirow{2}{*}{ Los Angeles } & 672.062 & $16 \%$ & & 546,031 & $15 \%$ \\
Miami & 1755.866 & $42 \%$ & & $1,596,407$ & $44 \%$ \\
New York & 678.936 & $16 \%$ & & 683,646 & $19 \%$ \\
Houston & 847.545 & $20 \%$ & & 705,873 & $19 \%$ \\
Total 4 países & $3,954.409$ & $95 \%$ & & $3,531,957$ & $97 \%$ \\
\hline
\end{tabular}

*El total no suma $100 \%$ porque otros puertos reciben el restante de las importaciones de frijol. Fuente: USA trade on-line. 
importado de estos países. Las importaciones provenientes de Guatemala y Honduras han sido relativamente constantes en los últimos años, mientras que las importaciones de frijol provenientes de Nicaragua han aumentado en los últimos tres años. Desde 1994, EE.UU han importado más frijoles de que lo que han exportado a El Salvador. En los últimos años, esta diferencia entre importaciones y exportaciones ha aumentado. El Salvador es el proveedor principal de frijoles centroamericanos a EE.UU.

\section{Frijoles rojos pequeños y frijoles negros}

Estos dos grupos de frijoles representan la gran mayoría de las importaciones estadounidenses de frijoles centroamericanos. Aunque se reportan otros grupos de frijoles centroamericanos, se conoce que al entrar los frijoles a EE.UU., muchas veces son clasificados en categorías incorrectas como mungo y vigna. En este trabajo todas esas categorías se agruparon como Nesoi, entendiéndose que son malas clasificaciones.

En los pasados 10 años, El Salvador ha llegado a ser un proveedor importante de frijoles negros a EE.UU. (Figura 2), mientras los datos obtenidos, muestran las exportaciones de Guatemala en este rubro tendientes a dis- minuir. El Salvador es el principal proveedor en el mercado de frijoles, y ellos son responsables de más de la mitad de las importaciones de EE.UU. de frijoles rojos pequeños y frijoles negros provenientes de Centro América. Nicaragua ha aumentado sus exportaciones para llegar a ser el segundo proveedor más importante en este mercado (Figura 3).

\section{Descripción de sub-sector de frijoles centroamerica- nos en EE.UU}

Este sub-sector consiste en la combinación vertical de actividades asociadas con la producción y mercadeo del producto. La Figura 4 muestra los participantes asociados en este sub-sector.

\section{Descripción de participantes}

\section{Empresas productoras}

Estos son grandes productores de frijol que además tienen capacidad para comercializar su frijol con grandes mayoristas o directamente con empresas en el exterior. Algunos de estas empresas productoras, comercian directamente con los importadores en EE.UU. Algunas
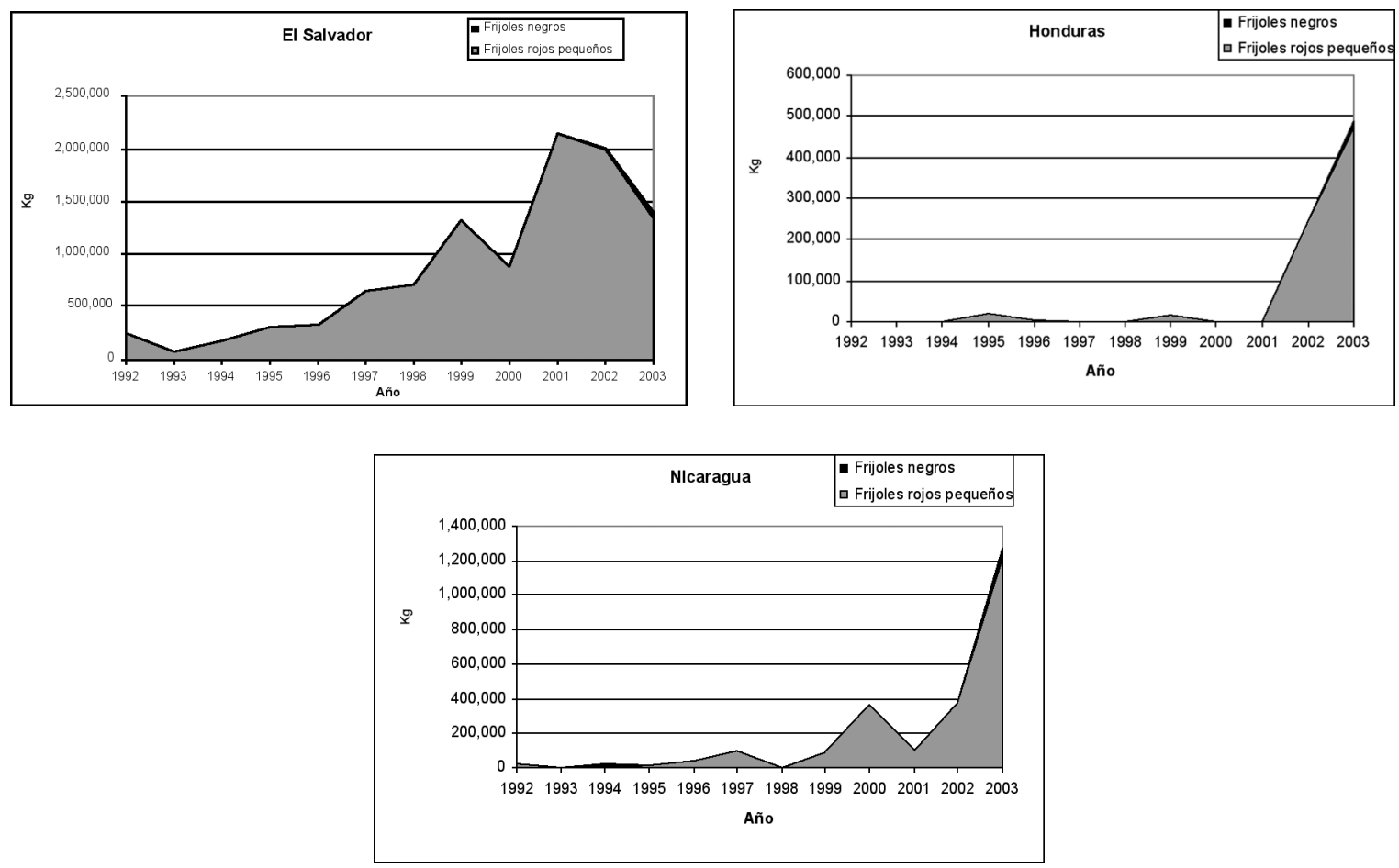

Figura 2. Cantidad (kg) de importaciones estadounidenses de frijoles rojos y negros provenientes de El Salvador, Honduras y Nicaragua por país, 1992-2003. Fuente: USA Trade on-line. 

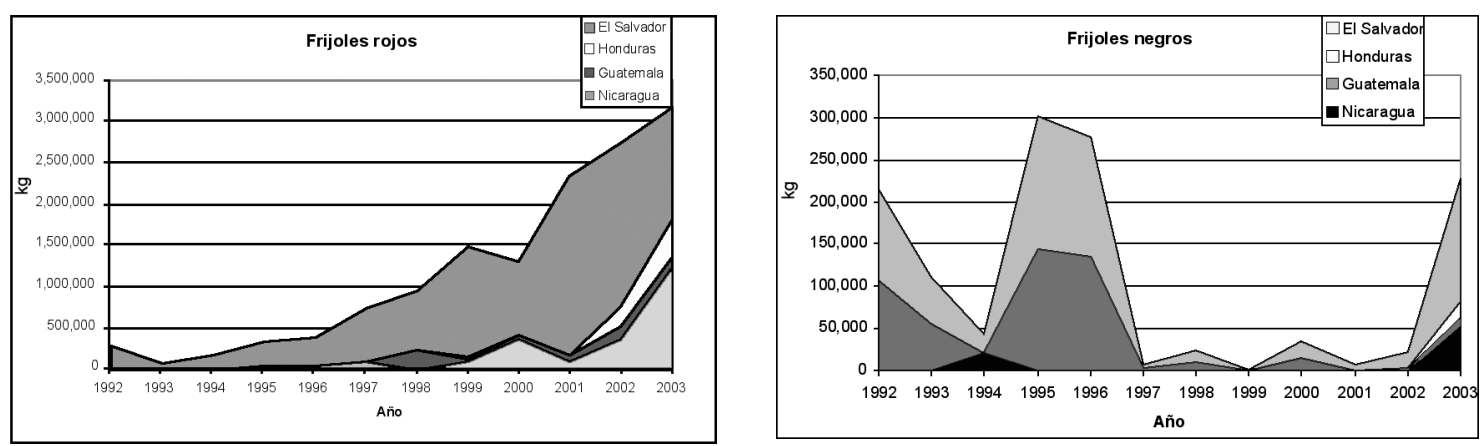

Figura 3. Cantidad $(\mathrm{kg})$ de importaciones estadounidenses de frijoles rojos y negros provenientes de El Salvador, Guatemala, Honduras y Nicaragua, por tipo de frijol, 1992-2003. Fuente: USA Trade online.

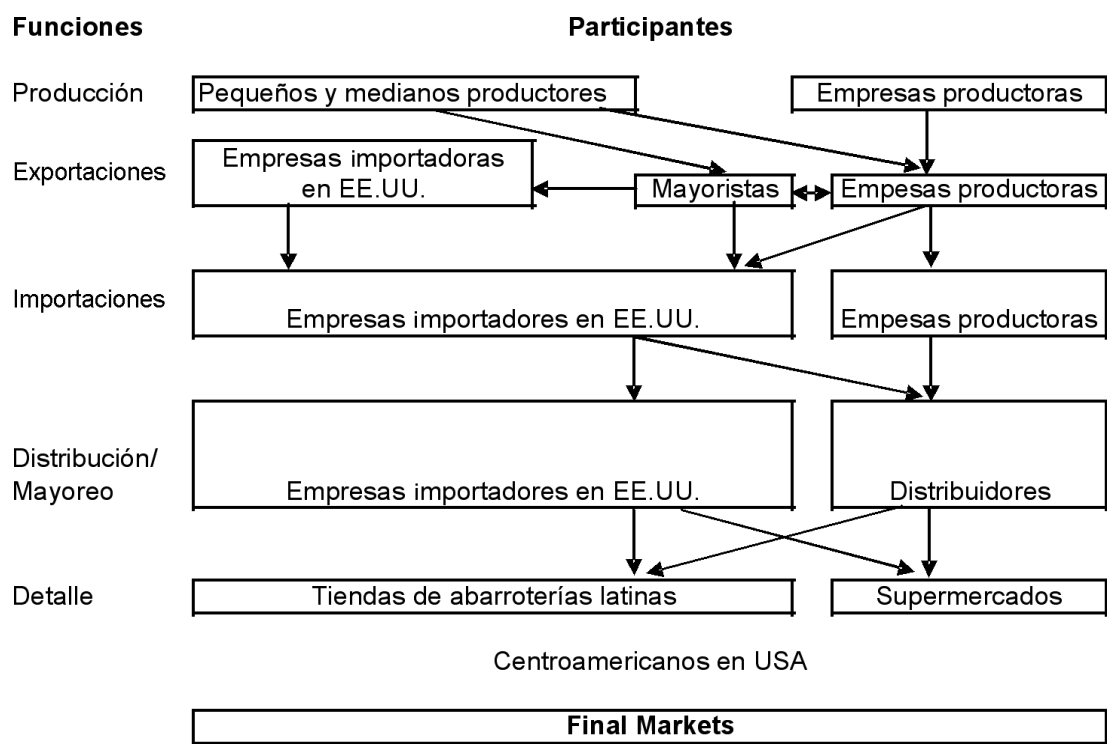

Figura 4. Mapa del sub-sector de frijoles centroamericanos en EE.UU. 2003. Elaboración del autor.

empresas productoras ya tienen firmas representantes en EE.UU para importar sus propios frijoles. En el último caso, estas firmas pueden realizar algún tipo de distribución de sus propios productos o usan distribuidores en EE.UU. Arrocera San Francisco (ASF), en El Salvador, es un ejemplo de estas últimas firmas.

\section{Mayoristas en Centro América}

Este grupo compra frijoles en Centro América directamente de los productores o de otros intermediarios. Algunos de ellos piden características específicas de calidad. Los mayoristas es este sub-sector exportan frijoles a EE.UU donde los importadores compran el producto.

\section{Importadores}

Varias personas de origen centroamericano en EE.UU. son dueños de la mayoría de las firmas que importan frijoles provenientes de Centro América. Ellos importan frijoles al granel (generalmente en bolsas de $46 \mathrm{~kg}$ ) o importan frijoles ya empacados en bolsas plásticas listos para ser vendidos al detalle. Las empresas importadoras están localizadas en las ciudades con las poblaciones más altas de centroamericanos en EE.UU, especialmente en Los Ángeles, Miami, Houston y New York. Estas empresas importadoras suelen tener representantes en Centro América y se abastecen ya sea de firmas en Centro América que cultivan frijoles y/o compran frijoles de mayoristas en Centro América o de sus 
representantes que consiguen frijoles en la región. Estos frijoles centroamericanos vienen generalmente a EE.UU junto a otros productos alimenticios, en contenedores mandados por las firmas exportadores en Centro América. El costo de transporte por mandar un contenedor proveniente de Centro América a los EE.UU. es aproximadamente 2.500 a 3.000 dólares. En algunos casos los importadores distribuyen también los frijoles en EE.UU. Hay alrededor de 30 empresas conocidas en EE.UU que importan frijoles de Centro América. En Los Ángeles se localiza la mayoría de estas firmas. Houston, Washington, New York y Chicago también tienen empresas que importan frijoles provenientes de El Salvador y Guatemala (Cabrera 2003).

\section{Distribuidores}

Los distribuidores compran frijoles de los importadores en EE.UU. Ellos proveen a abarroterías y supermercados con el producto. Los distribuidores tienen una cantidad diversa de productos étnicos que comercializan en estas tiendas detallistas.

\section{Abarroterías y supermercados étnicos}

Estas tiendas étnicas se localizan en áreas con alta concentración de hispanos en EE.UU. Varias de estas tiendas tienen una gran diversidad de productos centroamericanos. El español es el principal idioma hablado y las personas que trabajan en estos lugares son mayormente hispanos. Algunos hispanos que viven en EE.UU son los principales dueños de estas tiendas. Personas de origen asiático poseen también algunas de estas tiendas, especialmente en Los Ángeles. En estas áreas, estas tiendas estás generalmente cerca una de la otra y son relativamente pequeñas (entre 75 y $200 \mathrm{~m}^{2}$ ) comparadas con los supermercados en el área. Algunas tiendas latinas se han convertido en supermercados y tienen un espacio más grande para vender y una cantidad más grande de productos (un ejemplo de estos supermercados es Liborios Markets en Los Ángeles). En las visitas a las cinco ciudades escogidas, 30 abarroterías hispanas fueron localizadas y visitadas en las zonas con la más alta concentración de centroamericanos en estas ciudades. Se reunió información acerca de la disponibilidad y precios de más de 30 marcas de frijol que se venden especialmente a centroamericanos. La mayoría del frijol rojo pequeño centroamericano encontrado fue salvadoreño, algunas marcas de este frijol fueron también nicaragüenses y muy pocas fueron hondureñas.

Los Cuadros 5 y 6 muestran los precios promedios por kilo encontrados para tres tipos de frijoles (negros,
Cuadro 5. Los precios promedios por kilo de diferentes tipos de frijoles centroamericanos y no-centroamericanos encontrados en muestreos realizados en tiendas en Los Ángeles, San Francisco, Chicago, Miami y Washington D.C. 2004. Elaboración del autor.

\begin{tabular}{|c|c|c|c|c|c|}
\hline Tipo de frijol & $\begin{array}{l}\text { No. de } \\
\text { observ. }\end{array}$ & Media & $\begin{array}{c}\text { Desv. } \\
\text { estándar }\end{array}$ & Máximo & Mínimo \\
\hline Negro CAN & 11 & 3,17 & 0,47 & 4,5 & 1,52 \\
\hline Negro No-CAN & 36 & 1,96 & 0,25 & 4,32 & 1,08 \\
\hline $\begin{array}{l}\text { Total frijoles } \\
\text { negros }\end{array}$ & 47 & 2,24 & 0,39 & 4,5 & 1,08 \\
\hline Rojo CAN & 10 & 3,06 & 0,36 & 4,08 & 1,71 \\
\hline Rojo No-CAN & 24 & 1,98 & 0,10 & 2,36 & 1,54 \\
\hline $\begin{array}{l}\text { Total frijoles } \\
\text { rojos }\end{array}$ & 36 & 2,32 & 0,31 & 4,08 & 1,54 \\
\hline Rojos de seda & 51 & 3,48 & 0,27 & 4,67 & 1,71 \\
\hline $\begin{array}{l}\text { Observaciones } \\
\text { totales }\end{array}$ & 1,34 & & & & \\
\hline
\end{tabular}

rojos y rojos de seda). Dentro de los frijoles negros y rojos pequeños, se diferencia los frijoles etiquetados como centroamericanos, y los no etiquetados como centroamericanos. Los frijoles rojos de seda tuvieron precios en estas tiendas en promedio alrededor de 50\% más altos $(\$ 3.47 / \mathrm{kg})$ que los frijoles rojos pequeños regulares $(\$ 2.33 / \mathrm{kg})$. Los frijoles rojos centroamericanos tuvieron precios en promedio alrededor de $50 \%$ más alto $(\$ 3.06 / \mathrm{kg})$ que frijoles no-centroamericanos $(\$ 2.01 / \mathrm{kg})$.

Cuadro 6. Precios promedios por kilo de diferentes frijoles de América Central encontrados en muestreos realizados en tiendas en Los Ángeles, San Francisco, Chicago, Miami y D.C. por país específico. 2004.

\begin{tabular}{lccrrrr}
\hline \multirow{2}{*}{$\begin{array}{l}\text { Etiquetado } \\
\text { como: }\end{array}$} & \multicolumn{2}{c}{ Rojo } & \multicolumn{2}{c}{ Rojo de seda } & \multicolumn{2}{c}{ Total } \\
& N & Media & N & Media & N & Media \\
\hline Salvadoreño & 7 & 3,00 & 38 & 3,49 & 45 & 3,39 \\
Nicaragüense & 1 & 2,89 & 11 & 3,50 & 12 & 3,44 \\
Hondureño & 1 & 3,46 & 1 & 3,45 & 2 & 3,46 \\
\hline
\end{tabular}

Los frijoles negros centroamericanos tuvieron precios alrededor del $60 \%$ más alto $(\$ 3.17 / \mathrm{kg})$ que los frijoles negros no-centroamericanos $(\$ 1.96 / \mathrm{kg})$. Los precios promedios para frijoles rojos y rojos de seda no cambian mucho al compararlos entre países. 


\section{Consumidores finales}

Ciento catorce consumidores fueron encuestados en Washington D.C., Chicago, Miami, San Francisco y Los Ángeles, para obtener información acerca de sus preferencias en el consumo de frijol. Cerca del $90 \%$ de los centroamericanos encuestados dijeron que ellos compran frijoles (Cuadro 7). El consumo medio de los que dijeron que compran frijoles fue de $1,1 \mathrm{~kg}$ por hogar por semana. El hogar promedio tiene aproximadamente cuatro personas.

Cuadro 7. Frecuencia y porcentaje de respuestas a la pregunta del consumo o no de frijol en bolsa realizada a centroamericanos en EE.UU, por país específico. 2004.

\begin{tabular}{|c|c|c|c|c|c|}
\hline \multirow{3}{*}{$\begin{array}{c}\text { País de } \\
\text { de origen } \\
\text { encuestados }\end{array}$} & \multicolumn{4}{|c|}{ Compra frijoles en bolsa? } & \multirow{3}{*}{$\begin{array}{l}\text { Total } \\
\text { N }\end{array}$} \\
\hline & \multicolumn{2}{|r|}{ No } & \multicolumn{2}{|r|}{ Sí } & \\
\hline & $\mathbf{N}$ & $\begin{array}{c}\text { \% dentro de } \\
\text { país de } \\
\text { origen }\end{array}$ & $\mathbf{N}$ & $\begin{array}{c}\text { \%dentro de } \\
\text { país de } \\
\text { origen }\end{array}$ & \\
\hline El Salvador & 3 & 8,1 & 34 & 91,9 & 37 \\
\hline Guatemala & 2 & 8,3 & 22 & 91,7 & 24 \\
\hline Nicaragua & 6 & 18,2 & 27 & 81,8 & 33 \\
\hline Honduras & 3 & 15,0 & 17 & 85,0 & 20 \\
\hline Total & 14 & 12,3 & 100 & 87,7 & 114 \\
\hline
\end{tabular}

Los salvadoreños encuestados dijeron que sus hogares consumen cerca de $0,46 \mathrm{~kg}$ por semana de frijo- les rojos de seda de El Salvador, y cerca de 0,23 kg más de frijoles rojos pequeños y frijoles negros de El Salvador (Cuadro 8). En general, los centroamericanos encuestados dijeron consumir en promedio alrededor de $0,9 \mathrm{~kg}$ de frijol en sus hogares por semana (contando los que consumen y no consumen frijoles), de las cuales, alrededor de $0,46 \mathrm{~kg}$ es de frijol de su propio país y casi $0,23 \mathrm{~kg}$ es de otra parte de Centro América. Los hondureños son los que dijeron consumir la cantidad más pequeña de frijoles centroamericanos. Los guatemaltecos son los que consumen la cantidad más pequeña de frijoles de su propio país y los que consumen mayor cantidad de frijoles en lata. Esto puede ser debido a la preferencia de los guatemaltecos por frijoles negros y a la disponibilidad de frijoles negros en EE.UU. semejantes a los frijoles que ellos prefieren.

Varias personas que compran frijoles rojos pequeños fueron encuestadas acerca de cual era la característica más importante para ellos al momento de comprar los frijoles rojos en bolsa.

En esta pregunta, los encuestados tuvieron la oportunidad de escoger entre "el color" y "la procedencia" (el lugar del origen) de los frijoles, o para decir que ellos no tenían una preferencia entre esos dos atributos (Cuadro 9). Las personas escogieron "la procedencia" el doble de las veces que "el color" fue escogido. Esos resultados son parecidos a los resultados cuando esa pregunta es analizada por país específico. Luego de esto, se les pidió a las personas escoger entre cada una de esas características. Ellos pudieron escoger dentro de

Cuadro 8. Cantidad de frijoles consumidos ( $\mathrm{kg} / \mathrm{semana} / \mathrm{hogar}$ ) por centroamericanos encuestados en EE.UU, país de origen y por tipo de frijoles. 2004.

\begin{tabular}{|c|c|c|c|c|c|c|c|c|c|c|c|c|c|}
\hline \multirow{3}{*}{\multicolumn{2}{|c|}{ País d }} & \multirow[b]{3}{*}{ Total } & \multicolumn{11}{|c|}{ Frijoles } \\
\hline & & & \multicolumn{4}{|c|}{ De su país de origen } & \multicolumn{4}{|c|}{$\begin{array}{l}\text { De otro lugar de } \\
\text { Centroamérica }\end{array}$} & \multicolumn{3}{|c|}{ De cualquier otro lugar } \\
\hline & & & Rojo & Negro & $\begin{array}{c}\text { Rojo } \\
\text { de seda }\end{array}$ & Otro & Rojo & Negro & $\begin{array}{c}\text { Rojo } \\
\text { de seda }\end{array}$ & Otro & Rojo & Negro & Otro \\
\hline El Salvador & Media & 0,99 & 0,30 & 0,02 & 0,57 & 0 & 0 & 0 & 0 & 0 & 0,05 & 0,04 & 0,01 \\
\hline \multirow[t]{2}{*}{$\mathrm{N}=37$} & Est.* & & & & & & & & & & & & \\
\hline & Desv. & 1,69 & 0,50 & 0,15 & 0,75 & 0 & 0 & 0,04 & 0 & 0 & 0,24 & 0,14 & 0,07 \\
\hline \multirow{3}{*}{$\begin{array}{l}\text { Guatemala } \\
\mathrm{N}=24\end{array}$} & Media & 0,9 & 0 & 0,10 & 0 & 0 & 0,06 & 0,31 & 0 & 0,03 & 0,02 & 0,31 & 0,02 \\
\hline & Est. & & & & & & & & & & & & \\
\hline & Desv. & 1,69 & 0 & 0,23 & 0 & 0 & 0,15 & 0,53 & 0 & 0,10 & 0,09 & 0,43 & 0,06 \\
\hline \multirow{3}{*}{$\begin{array}{l}\text { Nicaragua } \\
N=33\end{array}$} & Media & 0,97 & 0,35 & 0 & 0,04 & 0 & 0,28 & 0 & 0 & 0 & 0,12 & 0,07 & 0,11 \\
\hline & Est, & & & & & & & & & & & & \\
\hline & Desv. & 1,69 & 0,79 & 0 & 0,24 & 0 & 0,48 & 0 & 0 & 0 & 0,28 & 0,20 & 0,50 \\
\hline \multirow{3}{*}{$\begin{array}{l}\text { Honduras } \\
\mathrm{N}=20\end{array}$} & Media & 0,57 & 0,16 & 0,01 & 0 & 0 & 0,11 & 0 & 0 & 0 & 0,14 & 0,11 & 0,05 \\
\hline & Est. & & & & & & & & & & & & \\
\hline & Desv. & 1,69 & 0,36 & 0,03 & 0 & 0 & 0,25 & 0 & 0 & 0 & 0,27 & 0,25 & 0,20 \\
\hline \multirow{3}{*}{$\begin{array}{l}\text { Total } \\
N=144\end{array}$} & Media & 0,89 & 0,23 & 0,03 & 0,20 & 0 & 0,11 & 0,07 & 0 & 0 & 0,08 & 0,12 & 0,05 \\
\hline & Est. & & & & & & & & & & & & \\
\hline & Desv. & 1,69 & 0,54 & 0,142 & 0,51 & 0 & 0,30 & 0,27 & 0 & 0,5 & 0,24 & 0,27 & 0,29 \\
\hline
\end{tabular}

*Est. Desv.=desviación estándar. 
Cuadro 9. Frecuencia y porcentaje de respuestas para la pregunta que considera las preferencias al comprar frijoles rojos de centroamericanos en EE.UU encuestados, por país de origen, 2004.

\begin{tabular}{|c|c|c|c|c|c|c|c|}
\hline \multicolumn{8}{|c|}{ Escoja la principal característica que usted busca al comprar frijoles rojos } \\
\hline \multirow[b]{2}{*}{$\begin{array}{l}\text { País de origen } \\
\text { de encuestados }\end{array}$} & \multicolumn{2}{|c|}{ Color del frijol } & \multicolumn{2}{|c|}{ Procedencia del frijol } & \multicolumn{2}{|c|}{$\begin{array}{c}\text { Indiferente entre } \\
\text { esas dos características }\end{array}$} & \multirow[b]{2}{*}{ Total } \\
\hline & $\mathbf{N}$ & $\begin{array}{c}\text { \% dentro de } \\
\text { país de origen }\end{array}$ & $\mathbf{N}$ & $\begin{array}{c}\% \text { dentro de } \\
\text { país de origen }\end{array}$ & $\mathbf{N}$ & $\begin{array}{c}\text { \% dentro de } \\
\text { país de origen }\end{array}$ & \\
\hline El Salvador & 10 & 33,3 & 17 & 56,7 & 3 & 10,0 & 30 \\
\hline Nicaragua & 4 & 15,4 & 15 & 57,7 & 7 & 26,9 & 26 \\
\hline Honduras & 4 & 44,4 & 4 & 44,4 & 1 & 11,1 & 9 \\
\hline Total & 18 & 27,7 & 36 & 55,4 & 11 & 16,9 & 65 \\
\hline
\end{tabular}

las opciones siguientes: dentro de "la procedencia" ellos pudieron escoger entre "de su país del origen", "de otro lugar en Centro América", "de dondequiera más en el mundo" o "no preferencia". Más del $80 \%$ dijo que ellos prefieren que los frijoles vengan de su país de origen (Cuadro 10).

\section{Dinámicas dentro del sub-sector}

Los frijoles salvadoreños son los que se encontraron más comúnmente en los recorridos. Las firmas que distribuyen estos frijoles pertenecen generalmente a centroamericanos en EE.UU. Ellos distribuyen estos frijoles, principalmente a niveles regionales, enfocando al mercado salvadoreño en Los Ángeles, Washington D.C y Houston, especialmente. Los frijoles de Río Grande se encontraron en San Francisco, D.C. y Los Ángeles. Los frijoles de La Migueleña se encontraron en D.C y Chicago. Los frijoles de la empresa Goya fueron los únicos que se encontraron en cada ciudad que se visitó. Goya tiene una presencia predominante en las abarroterías que se visitaron, teniendo una gran variedad de productos en estas tiendas. Goya tiene frijoles negros, frijoles rojos pequeños, rojos de seda y frijoles rojos etiquetados como centroamericanos. Goya enfoca diferentes nichos en diferentes ciudades.

Considerando que los frijoles que estos comerciantes salvadoreños traen a EE.UU no son solamente frijoles salvadoreños sino también de Honduras y Nicaragua, algunos de estos productos enfocan también a los mercados hondureños y nicaragüenses, al etiquetar algunos de sus frijoles como hondureños o nicaragüenses. Algunos frijoles nicaragüenses se encontraron en abarroterías latinas en Miami y San Francisco.

En general en este sub-sector hay dos canales claramente identificables por los que se comercializa el frijol: 1) el canal orientado por los importadores, 2) el canal orientado por los exportadores.

\section{Canal orientado por los importadores}

Este canal está constituido por el grupo de los importadores que tienen alguna clase de canal de distribución regional o interregional en EE.UU. Las empresas

Cuadro 10. Frecuencia y porcentaje de respuestas para la pregunta que considera las preferencias en la procedencia del frijol de centroamericanos en EE.UU encuestados por país de origen. 2004.

\begin{tabular}{|c|c|c|c|c|c|c|c|}
\hline \multirow[b]{3}{*}{$\begin{array}{l}\text { País de origen } \\
\text { de encuestados }\end{array}$} & \multicolumn{6}{|c|}{ Procedencia preferida al comprar frijoles rojos } & \multirow[b]{3}{*}{ Total } \\
\hline & \multicolumn{2}{|c|}{ Su país de origen } & \multicolumn{2}{|c|}{$\begin{array}{c}\text { Cualquier lugar del } \\
\text { mundo }\end{array}$} & \multicolumn{2}{|c|}{$\begin{array}{l}\text { No preferencia } \\
\text { en esta pregunta }\end{array}$} & \\
\hline & $\mathbf{N}$ & $\begin{array}{c}\% \text { dentro de } \\
\text { país de origen }\end{array}$ & $\mathbf{N}$ & $\begin{array}{c}\text { \% dentro de } \\
\text { país de origen }\end{array}$ & $\mathbf{N}$ & $\begin{array}{c}\% \text { dentro de } \\
\text { país de origen }\end{array}$ & \\
\hline El Salvador & 24 & 80,0 & & & 6 & 20,0 & 30 \\
\hline Nicaragua & 22 & 84,6 & & & 4 & 15,4 & 26 \\
\hline Honduras & 6 & 85,7 & 1 & 14,3 & & & 7 \\
\hline Total & 52 & 82,5 & 1 & 1,6 & 10 & 15,9 & 63 \\
\hline
\end{tabular}


Río Grande y Dubon \& Sons son ejemplos de estas firmas. Estas firmas están establecidas en EE.UU, tienen productos alimenticios que son enfocados al mercado centroamericano en EE.UU., especialmente al mercado salvadoreño. En este grupo existen también pequeñas empresas que han comenzado a traer frijoles de $\mathrm{Ni}$ caragua y Honduras para distribuirlos en canales regionales, por ejemplo en el área de Miami. Estos grupos obtienen frijoles de mayoristas o de sus propios representantes en Centro América y los exportan a sus firmas en EE.UU. Ellos distribuyen principalmente estos frijoles regionalmente; algunas firmas como Río Grande los distribuyen en varias regiones. En otros casos, estas firmas utilizan otras firmas especializadas en la distribución de productos étnicos. Estos productos se colocan en abarroterías étnicas principalmente, en zonas con alta concentración de centroamericanos. La empresa Goya es un ejemplo excepcional de este canal, ya que presenta una marcada integración vertical, poseyendo un extenso canal de distribución a nivel nacional y obteniendo el producto directamente en CA.

\section{Canal orientado por los exportadores}

El segundo grupo consiste en firmas en CA que cultivan y/o comercializan frijoles y han comenzado a exportar a EE.UU. Estas firmas compran también frijoles de otros productores en CA y de mayoristas y exportan sus productos principalmente a distribuidores en EE.UU. Algunas de estas firmas tienen representantes en EE.UU que importan estos frijoles y los entregan a distribuidores, por ejemplo Arrocera San Francisco. Muchas de estas firmas dijeron que la principal limitante para aumentar sus exportaciones es obtener información acerca de donde se encuentra el mercado y qué distribuidores estarían interesados en sus productos.

\section{Principales limitaciones y oportunidades para ex- pandir exportaciones en este sub-sector}

\section{Limitaciones y amenazas}

Algunas de las limitaciones expresadas por exportadores potenciales en Centro América son: 1) falta de información sobre contactos con importadores/distribuidores en EE.UU. que puedan mantener una relación segura y continua con ellos; 2) los sistemas actuales de cobranza por venta de productos en EE.UU. (cobro se puede realizar sólo hasta después de varias semanas ya que los importadores esperan vender algo de la mercadería antes de pagar por ella) hace que varias empresas vendan sus frijoles FOB en Centro América; 3) problemas para cumplir los requisitos de volumen y la consis- tencia en la calidad y tiempo. Algunas de las limitaciones expresadas por importadores en EE.UU son: 1) la variabilidad en la calidad del producto obtenido en Centro América; 2) los importadores pequeños dijeron que ellos tienen problemas para entrar al mercado de abarroterías ya que muchas veces ellos son suplidores nuevos de un producto nuevo; 3) falta de información sobre mercados nuevos donde puedan vender su producto; 4) encontrar exportadores confiables que puedan suministrar los volúmenes necesarios en el tiempo necesario.

\section{Oportunidades}

En general, los centroamericanos prefieren los productos de su país y están dispuestos a pagar un precio "premium" por esos productos. Algunos importadores de frijoles piensan que hay un mercado potencial para frijoles centroamericanos, especialmente para frijoles hondureños y nicaragüenses. Estos importadores expresaron también que obtener la información acerca de distribución de los centroamericanos en EE.UU. les ayudaría a aumentar sus ventas de frijol. Hay muchos distribuidores en EE.UU. que venden productos alimenticios al mercado étnico latino. Algunos de estos distribuidores están interesados en entrar al mercado étnico de alimentos centroamericanos. Estos distribuidores necesitan información sobre la ubicación de los centroamericanos en EE.UU. y sobre los exportadores centroamericanos confiables que puedan cumplir los requisitos de volumen, calidad y consistencia. Estos requisitos varían entre diferentes distribuidores.

El canal orientado por importadores pareciera tener el mejor escenario para crecer. Los importadores en EE.UU. tienden a aumentar la coordinación vertical debido a la inconsistente calidad del producto de algunos comerciantes en Centro América y a la falta del acceso a este mercado para agricultores en esta región. Algunos importadores en EE.UU. han comenzado a crear acuerdos con agricultores y empresas en Centro América (Cabrera 2003).

\section{Limitaciones del estudio}

La limitación más grande en este estudio es el número real de encuestas que se realizaron a consumidores. Fue muy difícil, logísticamente hablando, alcanzar un número más grande de consumidores encuestados. Esta limitación se podría reducir, en estudios futuros parecidos, trabajando de cerca con las embajadas y consulados centroamericanos para obtener un número mayor de encuestas a consumidores. 


\section{Implicaciones políticas}

Nuevas iniciativas son necesarias para ayudar a los exportadores centroamericanos a identificar nuevos mercados en EE.UU. y para asegurar que los productores agrícolas pequeños también se beneficien del crecimiento potencial de las exportaciones en este sub-sector.

Primeramente, los consulados y especialmente los organismos para fomentar exportaciones y las embajadas de los países centroamericanos podrían ayudar a obtener la información necesaria para cuantificar la demanda de productos alimenticios centroamericanos en los mercados étnicos en EE.UU. Estas organizaciones reciben centenares de centroamericanos cada semana en sus oficinas, por lo que podrían ayudar a evaluar las preferencias de los consumidores centroamericanos a través de recolección de información vía encuestas. Las embajadas podrían ser también la conexión entre importadores y/o distribuidores en EE.UU. y exportadores centroamericanos, proveyendo a los importadores/distribuidores en EE.UU. con información sobre los exportadores centroamericanos que permita crear un ambiente de confianza en las posibles relaciones comerciales entre los grupos en EE.UU. Otros países latinoamericanos han optado por usar sus oficinas gubernamentales para respaldar a los exportadores de sus países en la búsqueda de mercados en EE.UU. El gobierno salvadoreño ha trabajado de cerca con exportadores salvadoreños y contactos en EE.UU. para aumentar la presencia de productos salvadoreños en EE.UU. Esto es un ejemplo exitoso del que los otros países centroamericanos podrían obtener valiosa información.

Segundo, para aumentar la participación de los pequeños productores agrícolas en este sub-sector se necesita investigar diferentes políticas que permitan a los pequeños productores obtener el necesario capital físico, financiero, organizativo o humano. Las políticas que ayuden a estos pequeños productores de frijol a almacenar y comercializar como grupo, además de facilitar oportunidades de negocio entre importadores en EE.UU. y pequeños productores organizados en Centro América, son oportunidades de intervención potenciales.

\section{RECONOCIMIENTOS}

Esta publicación ha sido posible a través del apoyo del Programa Bean/Cowpea Collaborative Research Support Program (CRSP), auspiciado por la Agencia para el Desarrollo Internacional de los Estados Unidos de Norteamérica (United States Agency for International Development, USAID), Donación No. GDG-G-00-
02-00012-00. Las opiniones expresadas por los autores en este documento no reflejan necesariamente los puntos de vista de USAID.

Agradecemos al Dr. Juan Carlos Rosas de la Escuela Agrícola Panamericana, Zamorano, Honduras, por su colaboración en la edición de este documento.

\section{LITERATURA CITADA}

BATRES, S; JENSEN, H; BRESTER, G. 2003. Salvadoran consumption of ethnic foods in the United States. Journal of Food Distribution Research 34 (2): 1-16.

CABRERA, O. 2003. Nuevo cohete comercial rumbo a EE.UU. (en línea). El Diario. San Salvador. Consultado 12 jun. 2003. Disponible en: www.elsalvador. com/noticias/2003/2/12/negocios/ negoc3.html.

FOOD MARKETING INSTITUTE. 2002. U.S. Hispanics: insights into grocery shopping preferences \& attitudes, executive summary (en línea). Food Marketing Institute, USA. Consultado 5 jun. 2003. Disponible en: http://fmi.org/pub/summaries/hispanicexecsummary.pdf

HUMPHREYS, J. 2003. The multicultural economy 2003, America's minority buying power. Georgia Business and Economic Conditions. 63(2):1-26.

LUCIER, G; LIN, B; ALLSHOUSE, J; KANTOR, L. 2000. Factors affecting dry bean consumption in the United States (en línea). Washington, D.C. Economic Research Service/USDA. Consultado 15 ene. 2003. Disponible en: http://www.ers.usda.gov/briefing/Consumption/ gallery/DryBeanConsumption.pdf

MINISTERIO DE ECONOMÍA DE EL SALVADOR. 2003. Estudio de mercado de productos étnicos en Estados Unidos: caso de estudio frijol rojo salvadoreño. San Salvador. Ministerio de Economía de El Salvador. Consultado 15 ene. 2003. Disponible en: http://www. minec.gob.sv/media/downloads/Estudio_de_Mercado_ de_Frijoles_de_Seda.pdf

OFICINA DEL CENSO DE EE.UU. 2000. Census 2000 Basics (en línea). Consultado 2 set. 2003. Disponible en: http://www.census.gov/mso/www/c2000basics/chapter4.htm

OFICINA DEL CENSO DE EE.UU. 2001. PCT006. Hispanic or Latino by specific origin - Universe: total population (en línea). Consultado 4 set. 2003. Disponible en: http://factfinder.census.gov/servlet/DTTable?_bm= 
y\&-geo_id=D\&-ds_name=D\&_lang=en\&-mt_name=ACS_C2SS_EST_G2000_PCT006

USA trade on-line. 2003. Base de datos de comercio exterior de EE.UU. (en línea). Consultado 18 ene. 2003. Disponible en: www.usatradeonline.gov
UNITED STATUS DEPARTMENT OF AGRICULTURE (USDA). 1998. Continuing survey of food intakes by individuals (CSFII). Washington, D.C. Agricultural Research Service/USDA. 